\title{
Automatic discrimination of laughter using distributed sEMG
}

\author{
S. Cosentino, S. Sessa, W. Kong, D. Zhang, A. Takanishi \\ Faculty of Science and Engineering, \\ Waseda University \\ Tokyo, Japan
}

\begin{abstract}
Laughter is a very interesting non-verbal human vocalization. It is classified as a semi voluntary behavior despite being a direct form of social interaction, and can be elicited by a variety of very different stimuli, both cognitive and physical. Automatic laughter detection, analysis and classification will boost progress in affective computing, leading to the development of more natural human-machine communication interfaces. Surface Electromyography (sEMG) on abdominal muscles or invasive EMG on the larynx show potential in this direction, but these kinds of EMG-based sensing systems cannot be used in ecological settings due to their size, lack of reusability and uncomfortable setup. For this reason, they cannot be easily used for natural detection and measurement of a volatile social behavior like laughter in a variety of different situations. We propose the use of miniaturized, wireless, dry-electrode sEMG sensors on the neck for the detection and analysis of laughter. Even if with this solution the activation of specific larynx muscles cannot be precisely measured, it is possible to detect different EMG patterns related to larynx function. In addition, integrating sEMG analysis on a multisensory compact system positioned on the neck would improve the overall robustness of the whole sensing system, enabling the synchronized measure of different characteristics of laughter, like vocal production, head movement or facial expression; being at the same time less intrusive, as the neck is normally more accessible than abdominal muscles. In this paper, we report laughter discrimination rate obtained with our system depending on different conditions.
\end{abstract}

Keywords-laughter; EMG; electromyography; affective computing; laughter computing.

\section{INTRODUCTION}

Laughter is an universally recognizable non-verbal human social communication signal [1], and has been object of study in various fields, from psychology and psychiatry [2]-[5], to medicine and neuroscience [6]-[9], and, more recently, computer science [10], [11].

In fact, a thorough understanding of laughter as one of the mechanisms at the basis of natural human social interaction and communication would lead to a dramatic improvement of human-machine communication and possible new applications.

However, while verbal communication is usually direct, and it is processed on a conscious level, non-verbal

This research has been supported by the JSPS Grant-in-Aid for Young Scientists (Wakate B) [25750259] and [15K21437], and MEXT, Japan. It has been also partially supported by a grant by STMicroelectronics, which also provided the core sensors and the microcontrollers.

\author{
N. Bianchi-Berthouze, \\ UCL Interaction Centre, \\ University College of London (UCL) \\ London, UK
}

communication is usually processed at an unconscious level [12], complementing and enriching direct interchanges carrying additional information on the emotional state of the subjects. Moreover, non-verbal social communication signals cannot be voluntarily controlled, performed or suppressed [1], and are multimodal, usually involving the coordination of different groups of muscles, and the alteration of several physiological parameters [13]. The ability to process and understand non-verbal communication feedback is paramount to calibrate the level of exchange among interacting partners, and to orient the conversation in the desired direction, ensuring that the message is received according to one's endeavor.

There is already a vast body of literature on laughter detection and classification, using standard noninvasive methods like audio and video data processing [14]-[22]. However, there are situations in which audio and video systems cannot be used or cannot be considered reliable, e.g. long-term laughter monitoring, multiple interaction, or noisy environment. The aim of this paper is then to present the development of a new ecological EMG-based sensor system for measuring, recording and classifying laughing real-time during prolonged social natural interaction activities. This system could be used as an alternative or as an auxiliary laughter detection and classification system in those situations in which other less invasive systems cannot be used. In addition, this system could be used to clarify the muscles activities during laughter and help drawing a physical and physiological model of laughter, as well as building a body action code system for muscles-emotion.

The paper is organized as following: Section II describes the theoretical and practical grounds on which our system and sensing methodology are based, the choices in design and implementation, and the experiment setup to collect laughter data among other vocalization; Section III describes the results of the experiments; Section IV discusses those results; and Section V presents the conclusions and future work.

\section{MATERIAL AND METHODS}

Several muscles are simultaneously activated to produce laughter: the ones related to sound production (respiratory muscles, larynx apparatus); the ones related to expression (facial muscles); and some other as byproduct of the intensity of the emotional arousal (arm gesturing, posture alterations) [13]. 
Different types of sensor systems have been used to detect and quantify laughing, depending on the measured parameters [23]. The most ecological systems are audio and video based, but they present several drawbacks: with audio systems it is difficult to capture individual data during interactions among multiple subjects, and video data is normally not sufficient to properly detect and classify laughing. Moreover, both these methods might raise privacy issues. Motion capture systems, like Vicon, also are a valid alternative to record reliable data in an ecological environment, but those systems are normally very expensive, need a long setup and the markers must always be in sight. On the other hands, several studies successfully detected and measured laughing data using EMG in clinical settings.

\section{A. $E M G$}

We decided to use the EMG prototype designed by our group named WB-EMG (Waseda Bioinstrumentation system EMG). Our sensor specifications, shown in Fig. 1, were selected based on ISEK [24] and SENIAM standards [25]. The surface area of active electrodes is a very important factor and overall size of electrodes is critical for a good Signal Noise Rejection (SNR) [26]. The sensor unit uses electrodes of cylindrical shape of $2 \mathrm{~mm}$ height and $6 \mathrm{~mm}$ diameter, with a rounded edge of $0.5 \mathrm{~mm}$ radius, placed along a line with an inter-electrode distance (IED) of $10 \mathrm{~mm}$, as shown in Fig. 1. Two of the electrodes are coupled electrodes for EMG local recording, whilst the third one is for reference, removing the need for a separated reference electrode. We also used several techniques to obtain an appropriate AD coupling, and common mode noise rejection as described in [27].

\section{B. Criteria to Select the Best Electrodes Position}

Beyond the fact that there is a growing literature showing that EMG can be used to discriminate between emotional states [28], [29], several studies confirmed the validity of EMG to detect laughter (see Fig. 2). Agostoni [30], measured respiration phases, muscle activation and transthoracic pressures with invasive EMG and a gastric balloon, and was able to classify laughing from other expiratory activities like coughing, and singing. Luschei [31] and Titze [32], using invasive intramuscular EMG, analyzed the dynamics of larynx during laughter. Kimura [33], and Kimata [34] successfully measured laughter with non-invasive sEMG on the xyphoid process, a traditional position to measure diaphragmatic activities for clinical respiration measurements. Strohl, [35] and Hoit, [36], instead, using sEMG on abdominal and thoracic

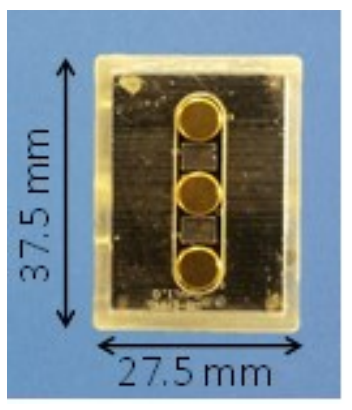

\begin{tabular}{lc}
\hline Parameter & Value \\
\hline Gain & 1400 \\
Weight & $13.4 \mathrm{~g}$ \\
Sampling Rate & $1 \mathrm{KHz}$ \\
Resolution & $12-\mathrm{bit}$ \\
Lower cutoff frequency & $20 \mathrm{~Hz}$ \\
Higher cutoff frequency & $450 \mathrm{~Hz}$ \\
CMRR & $>90 \mathrm{~dB}$ \\
\hline
\end{tabular}

Fig. 1. WB-EMG unit and its principal characteristics muscles to discriminate several respiratory and non-respiratory activities, found that better data are acquired on lower abdominal muscles. Following their lead, Fukushima [37] and Cosentino et al. [38] preferred abdominal muscles, measuring laughter with sEMG on the upper obliquus and Rectus Abdominis.

However, even if sEMG are less invasive, they might be uncomfortable to wear due to the required skin preparation, and not convenient due to the position. Also, depending on the position, they might be integrated in a more robust multisensory system. So we propose an EMG based system to detect, measure, and classify laughter on the neck, which is a position generally free and easily accessible, and where the sEMG can be integrated with a microphone to detect also the sound production associated with laughter. The full system is shown in Fig. 3.

Following the lead of Luschei and Titze, we chose to measure the electromyographic activity of the larynx and

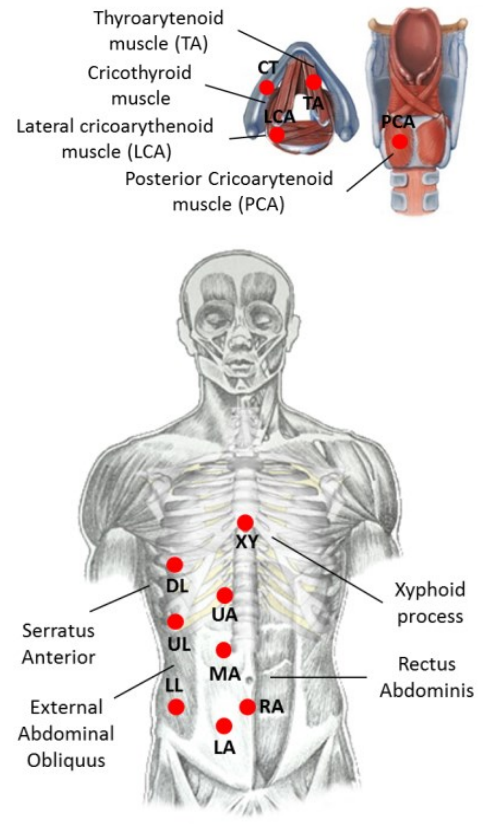

(a) Larynx activity

Luschei: all

Titze: LCA, TA, PCA

\section{(b) Respiration activity}

Strohl: UL, LL, MM

Hoit: UL, LL, UA, MA, LA

Kimura: UL, XY, RA

Kimata: XY

Fukushima: UL

Cosentino: UL, XY, RA

Fig. 2. EMG electrodes positions for laughter measurement

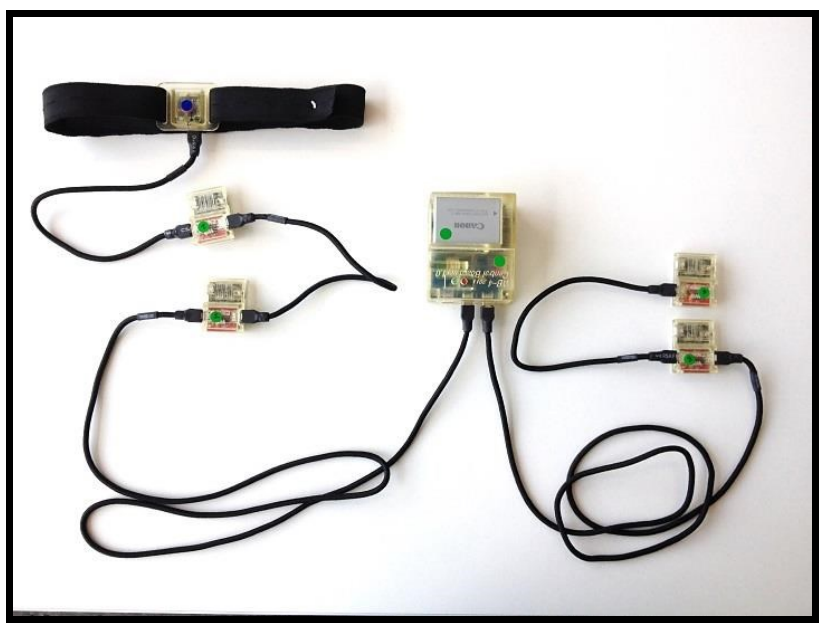

Fig. 3. WB-EMG sensor system 
surrounding muscles during various vocalizations and respiratory activities. The major difference is that we will not be able to measure electromyographic activity of specific muscles of the larynx, because those muscles are too deep and small for our EMG-based system. For this reason, the purpose of the preliminary study is detecting and classifying electromyographic mixed patterns related to different physiological activities.

\section{Experimental Setup}

The experiments were carried out at University College of London with ten healthy volunteers, of several nationalities, 4 males and 6 females, average age of 34.3 years old (18 to 63 years old), with no invasive devices and without any risk for the health of the participants. The right to privacy of the subjects has been fully respected, the subjects have been extensively informed and asked for written consent to the experiment, also taking into account their age and their health conditions, according to the existing national and international laws and regulations. Permission from the responsible Ethical Committee has been requested and obtained prior to these experiments.

Four synchronized WB-EMGs connected via CAN bus acquired data at a sample rate of $1 \mathrm{kHz}$ (see Fig. 3). Two EMGs were placed underneath the subject's chin, over the sternocleidomastoid muscle using medical grade tape on the left (LS) and right (RS) side. Two EMGs were placed on the abdominal muscles (LL and LA), in the same place of previous successful studies, as control data stream. The sensors were interconnected with special elastic electrical cables, provided by Asahi Kasei fibers corporation, to maximize comfort. For data communication a Central Board polls all the sensors connected to the CAN bus, packages the data and transmits to the $\mathrm{PC}$ over Bluetooth. The data is recorded in the $\mathrm{PC}$ and postprocessed in MATLAB. Both electrodes and skin at the sites were cleansed with common alcohol gel before positioning the electrodes.

Following the work by McKeown et al. [39] on laughter elicitation techniques, six subjects watched humorous videos of their choice (video stimulus mode), while four subjects interacted casually with a close friend, telling humorous anecdotes, talking about funny experiences, or playing the yes/no game (interaction mode). Fig. 4 describe the full procedure. The subjects wore the sensors and sat upright on a chair in a comfortable room, alone and with a headset in the case of humorous video stimulus, or together with a friend in the case of humorous interaction. The subjects were also asked to produce several specific types of vocalizations, listed in the next section. The EMG electrode placement and signal quality was verified by having the subjects talk and move for a few seconds before recording.

\section{Data Labelling}

Five hours of data were collected. One of the researchers segmented manually the data according to the following classes of events:

- $\quad$ Talking / Reading

- Laughter
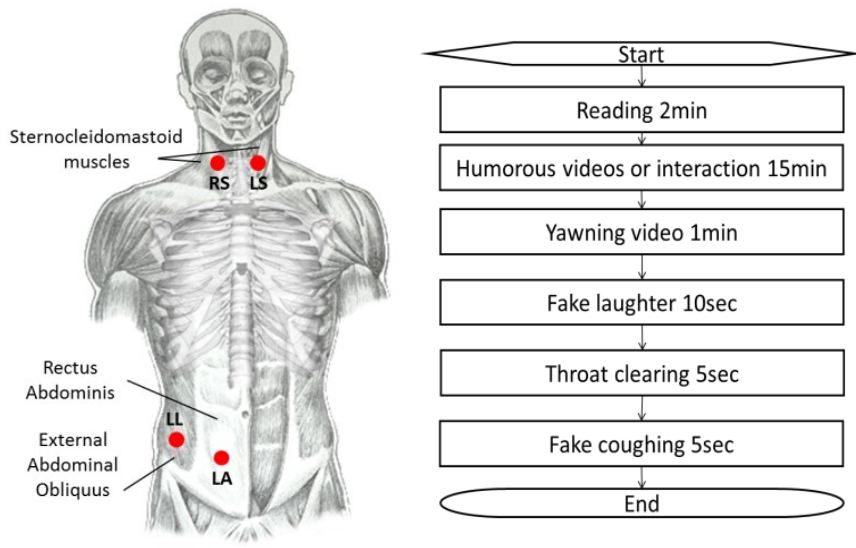

Fig. 4. Experiment setup.

- $\quad$ Fake laughter (Volitional laughter [40], [41] )

- Yawning

- Coughing

- Throat clearing

- Other action (none of the above)

The database with labeled data will be soon available online under the name of "UCL-WASEDA database".

\section{MODELING AND RESUlTS}

The aim of the study was to investigate the possibility to automatize the discrimination of laughter events from the other types of event listed in the previous section. The two conditions, video watching and friend interaction were modelled separately.

\section{A. Feature Extraction and Modeling}

The post processing has been performed with a MATLAB script that automatically loaded the raw EMG data files and arranged them into separate data sets according to the different action performed. A sliding window filter has been applied to each labeled EMG channel data (window step $=100 \mathrm{~ms}$, window length $=500 \mathrm{~ms}$ ), and features have been extracted to be used for discrimination between different vocal expressions.

Several candidate features, both in time and frequency domain, have been considered [42]. Among the considered features, the best results were obtained with the following subset of features:

- RMS (Root Mean Square) - the square root of the arithmetic mean of the squares of the samples

- Maximum Peak-to-Peak - the maximum excursion between highest and lowest amplitude value

in the time domain, and:

- Mean - the mean frequency of the windowed signal

- Mean power - mean of the values of the Power Spectral Density of the windowed signal

- Bandwidth - the difference between the upper and lower frequencies of the windowed signal 
TABLE I

EMG DATA STREAM RELIABILITY. $\mathrm{O}=$ ACCEPTABLE, BLANK $=$ SATURATED

\begin{tabular}{|c|c|c|c|c|}
\hline Subject & LS & RS & LA & LL \\
\hline 1 & O & O & & O \\
\hline 2 & O & & & \\
\hline 3 & & O & & O \\
\hline 4 & & O & & \\
\hline 5 & & O & & O \\
\hline 6 & O & O & & O \\
\hline 7 & O & O & & O \\
\hline 8 & O & O & & O \\
\hline 9 & O & O & O & O \\
\hline 10 & O & O & & O \\
\hline
\end{tabular}

in the frequency domain. The features were all normalized in range with respect to their respective maximum and minimum value obtained during all the set of experiments.

The same feature extraction and analysis have been performed on each different electrode site and the results have been compared.

Surprisingly, the two abdominal EMG data streams were highly variable depending on the subject and proved completely unreliable in this experiment, as opposed to the ones on the neck. This might be due to several causes, which have still to be investigated. For example, it might be that the average Body Mass Index (BMI) of the subjects (Japanese vs UK population) was much higher than in our previous experiments [38], so that the EMG on the abdominal region was not able to record proper muscle activation data. Also, it could be that the size of the muscles and the skin conductivity caused the saturation of the collected EMG signal. Constantly saturated signals cannot be used because give no information on muscle activation. In Table I the quality of the collected data stream for each location and for each subject is shown. Refer to Fig. 4 for the electrode site.

For this reason, the presented statistical analysis has been performed only on the neck EMG data streams, which were in any case our main target. Physiological activity classification has been performed on the basis of Principal Component Analysis (PCA) using two different classifiers, for performance comparison:

- Linear Discriminant (LD), for both binary (laughter/not laughter) and multi class classification

- $\quad$ Support Vector Machine (SVM), with a linear kernel, for specific binary classification laughter/not laughter.

For binary classification laughter/not laughter, spontaneous laughter vs other events, including volitional laughter, is intended. 10-fold Cross Validation technique [43] has been used to validate the results of the classification. Results were calculated for each single subject and globally, in a subjectindependent schema, on the full database. Depending on the classification conditions, classification results varied sensibly.

\section{B. Classification Results}

The performances of the classifiers have been measured in terms of Success rate, Error rate, Sensitivity and Specificity of the classifier with respect to Laughter class.

Sensitivity, also called the true positive rate (TP), or the recall rate in some fields, refers to the proportion of actual positive samples correctly classified as positive, in a binary classification test.

Specificity, also called the true negative rate (TN), refers to the proportion of negative samples correctly classified as negative.

If we identify as TP, FP (False Positive), TN, and FN (False Negative) the total numbers of true positives, false positives, true negatives, and false negatives, respectively, we can define:

$$
\begin{gathered}
\text { Sensitivity }=\frac{\mathrm{TP}}{\mathrm{TP}+\mathrm{FN}} \times 100 \% \\
\text { Specificity }=\frac{\mathrm{TN}}{\mathrm{FP}+\mathrm{TN}} \times 100 \% \\
\text { Correct Rate }=\frac{\mathrm{TP}+\mathrm{TN}}{\mathrm{TP}+\mathrm{TN}+\mathrm{FP}+\mathrm{FN}} \times 100 \%
\end{gathered}
$$

Classification results are shown in Table II, Table III, and Table IV. With "Global", intersubject classification is intended; all the data collected and labeled from all the subjects have been clustered according to their label in a single dataset for classification.

Results underlined are the ones worse than the statistical probability of correct rate by random classification (baseline $=0.142$ for 7 classes, baseline $=0.167$ for 6 classes, and baseline $=0.5$ for binary classification).

\begin{tabular}{|c|c|c|c|c|c|}
\hline Subject & Mode & $\begin{array}{c}\text { Data } \\
\text { samples }\end{array}$ & $\begin{array}{c}\text { Correct } \\
\text { Rate }\end{array}$ & Sensitivity & Specificity \\
\hline 1 & & 9398 & 0.607 & 0.084 & 0.906 \\
\hline 2 & & 11525 & $\underline{0.031}$ & 0.456 & 0.550 \\
\hline 3 & & 10604 & 0.234 & 0.081 & 0.956 \\
\hline 4 & I & 9837 & 0.311 & 0.170 & 0.923 \\
\hline 5 & I & 9335 & 0.210 & 0.256 & 0.861 \\
\hline 6 & & 13487 & $\underline{0.073}$ & 0.442 & 0.948 \\
\hline 7 & I & 13760 & 0.345 & 0.022 & 0.955 \\
\hline 8 & I & 7508 & $\underline{0.079}$ & 0.021 & 0.972 \\
\hline 9 & & 8458 & 0.426 & 0.009 & 0.989 \\
\hline 10 & & 3275 & 0.629 & 0.601 & 0.889 \\
\hline Global & & 97187 & 0.596 & 0.322 & 0.674 \\
\hline Average & & & 0.295 & 0.214 & 0.895 \\
\hline Max & & & 0.629 & 0.601 & 0.989 \\
\hline Min & & & 0.031 & 0.009 & 0.55 \\
\hline
\end{tabular}

TABLE II

Classification Results: LD 7 Classes 
The 7-class classifier (Table II) performs poorly on subjects 2, 6 and 8. Its average correct rate is, unsurprisingly, lower than the binary ones. However, an interesting finding is that its sensitivity and specificity are very unbalanced, sensitivity being definitely lower, at an average $20 \%$, whilst its specificity is higher, at an average of $90 \%$. Both binary classifiers (Table III and Table IV) perform rather poorly on data from subjects 2 , 3 and 9 , and have comparable global and average correct rate, around $65 \%$, and average sensitivity and specificity, respectively $50 \%$ and $63 \%$, more or less balanced.

TABLE III

ClASSIFICATION RESULTS: LD BINARY

\begin{tabular}{|c|c|c|c|c|c|}
\hline Subject & Mode & $\begin{array}{c}\text { Data } \\
\text { samples }\end{array}$ & $\begin{array}{c}\text { Correct } \\
\text { Rate }\end{array}$ & Sensitivity & Specificity \\
\hline 1 & & 9398 & 0.646 & 0.382 & 0.649 \\
\hline 2 & & 11525 & 0.505 & 0.563 & 0.505 \\
\hline 3 & & 10604 & $\underline{0.411}$ & 0.569 & 0.410 \\
\hline 4 & I & 9837 & 0.759 & 0.373 & 0.776 \\
\hline 5 & I & 9335 & 0.572 & 0.555 & 0.574 \\
\hline 6 & & 13487 & 0.899 & 0.465 & 0.923 \\
\hline 7 & I & 13760 & 0.584 & 0.375 & 0.592 \\
\hline 8 & I & 7508 & 0.631 & 0.358 & 0.664 \\
\hline 9 & & 8458 & $\underline{0.476}$ & 0.559 & 0.468 \\
\hline 10 & & 3275 & 0.749 & 0.771 & 0.748 \\
\hline \hline Global & & 97187 & 0.645 & 0.786 & 0.711 \\
\hline Average & & & 0.623 & 0.497 & 0.631 \\
\hline Max & & & 0.899 & 0.771 & 0.923 \\
\hline Min & & & 0.411 & 0.358 & 0.410 \\
\hline
\end{tabular}

TABLE IV

ClASSIFICATION RESUlTS: SVM BINARY

\begin{tabular}{|c|c|c|c|c|c|}
\hline Subject & Mode & $\begin{array}{c}\text { Data } \\
\text { samples }\end{array}$ & $\begin{array}{c}\text { Correct } \\
\text { Rate }\end{array}$ & Sensitivity & Specificity \\
\hline 1 & & 9398 & 0.745 & 0.338 & 0.748 \\
\hline 2 & & 11525 & $\underline{0.205}$ & 0.932 & 0.198 \\
\hline 3 & & 10604 & $\underline{0.339}$ & 0.606 & 0.337 \\
\hline 4 & I & 9837 & 0.820 & 0.219 & 0.847 \\
\hline 5 & I & 9335 & 0.530 & 0.639 & 0.519 \\
\hline 6 & & 13487 & 0.922 & 0.460 & 0.948 \\
\hline 7 & I & 13760 & 0.851 & 0.182 & 0.876 \\
\hline 8 & I & 7508 & 0.802 & 0.138 & 0.882 \\
\hline 9 & & 8458 & $\underline{0.205}$ & 0.894 & 0.134 \\
\hline 10 & & 3275 & 0.748 & 0.880 & 0.747 \\
\hline \hline Global & & 97187 & 0.674 & 0.829 & 0.516 \\
\hline Average & & & 0.617 & 0.529 & 0.624 \\
\hline Max & & & 0.922 & 0.932 & 0.948 \\
\hline Min & & & 0.205 & 0.138 & 0.134 \\
\hline
\end{tabular}

To understand better such findings, the confusion matrices have been calculated in the three classification cases, and they are shown in Table V, Table VI, Table VII, and Table VIII.

Table V and Table VI show that the two classifiers in the binary case laughter/not laughter seem to have comparable performances.

Table VII and Table VIII show the confusion matrix in the case of full classification with 7 classes, and classification when the non-specified data "other action" have been removed.

TABLE V

CONFUSION MATRIX: LD BINARY

\begin{tabular}{|c|c|c|c|}
\cline { 2 - 4 } \multicolumn{1}{c|}{} & \multicolumn{3}{|c|}{ Predicted class } \\
\hline \multirow{2}{*}{$\bar{*}$} & Subject 1 & Laughter & Not Laughter \\
\cline { 2 - 4 } & Laughter & 0.30 & 0.70 \\
\cline { 2 - 4 } & Not laughter & 0.34 & 0.66 \\
\hline
\end{tabular}

TABLE VI

CONFUSION MATRIX: SVM BINARY

\begin{tabular}{|c|c|c|c|}
\cline { 2 - 4 } \multicolumn{1}{c|}{} & \multicolumn{3}{c|}{ Predicted class } \\
\hline \multirow{2}{*}{} & Subject 1 & Laughter & Not Laughter \\
\cline { 2 - 4 } & Laughter & 0.32 & 0.68 \\
\cline { 2 - 4 } & Not laughter & 0.26 & 0.74 \\
\hline
\end{tabular}

TABLE VII

CONFUSION MATRIX: LD 7 CLASSES

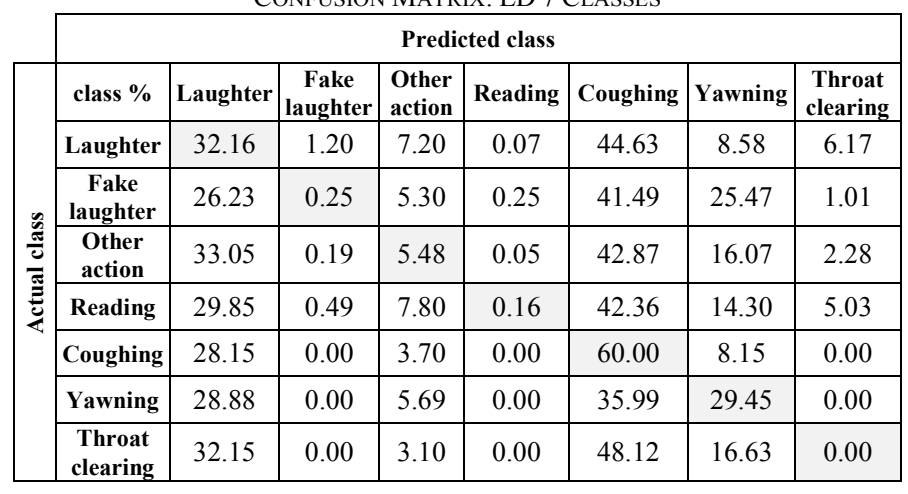

TABLE VIII

CONFUSION MATRIX: LD 6 CLASSES

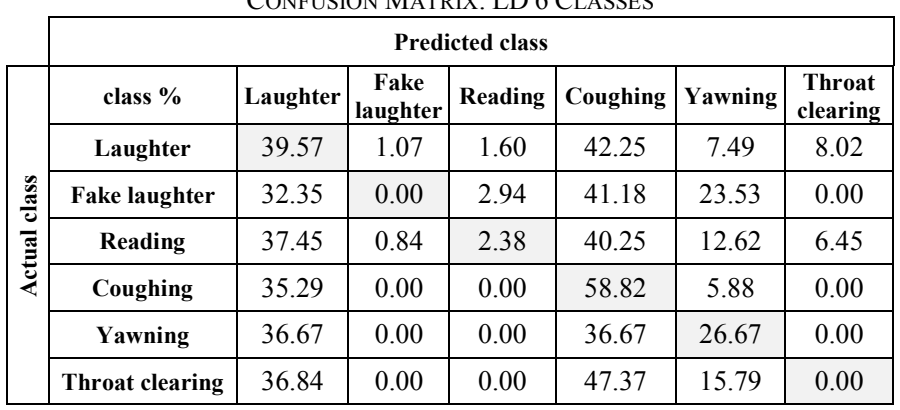




\section{DISCUSSION}

Discrimination between different vocalizations is possible for the neck EMG data based on the first three principal components, using a linear classifier, with a correct discrimination rate of $60 \%$, for a classification among 7 different classes, and of $65 \%$ for a binary classification laughter/not laughter, on the global database. Results are very similar using a SVM classifier for binary classification, with a correct classification rate of $67 \%$ on the global database. However, results for individual subjects are more consistent using the LD binary classifier, with a lower variance of correct rate, sensitivity and specificity among subjects.

Performances of the LD classifier change when used for binary classification, and for global action classification. In binary classification, sensitivity and specificity of both classifiers are balanced, at around $50 \%$. In global action classification, independently from the correct rate, the specificity of the classifier is generally high, while its sensitivity is low. The confusion matrix for the linear 7-class classifier is in line with global classification performance data: specificity is favored over sensitivity, and FN rate is much higher than TP rate for each single class.

Classification results and sensitivity definitely improve if we remove the "other action" data, which outnumber the other class data, skewing the data set, and also might include actions very different from each other thus difficult to categorize finely. Laughing is misclassified easily as coughing (42.25\%), and sometimes as yawning $(7.49 \%)$ and throat clearing $(8.02 \%)$. Reading/speaking is not easily recognized $(2.38 \%)$, and it is often misclassified either as laughter (37.45\%) or as coughing $(40.25 \%)$. Throat clearing and Fake laughter recognition rate is $0 \%$, and they are both misclassified with laughter, coughing, or yawing.

Interestingly, classification performances on subjects in different stimulus mode, interaction or video do not appear to be very different. The main difference in these modes is that subjects in interaction mode are interacting with someone, so "other action" class is mainly composed of talking, and random head and neck movement to maintain eye contact with the companion, while subjects watching videos are silent and mainly stationary. However, binary classification on subjects in interaction mode generally presents a lower sensitivity.

\section{CONCLUSIONS AND FUTURE WORKS}

In this paper we described a sEMG-based wearable sensor system to detect laughter on the neck and we presented the results of the experiment to validate the effectiveness of such system and of the proposed methodology. The results obtained by this experiment show that laughter may be detectable and measurable with non-invasive wearable sEMG sensors on the neck but better features extraction and tuning of the model is needed. In fact, the obtained results show that this system can recognize laughter from other different actions with a global correct discrimination rate of at least $64 \%$. These results, especially the ones obtained with the binary classifiers, compared with previous works in the field, using acoustic features [14]-[22], or other sEMG-based systems, [36], [44][49], are not particularly impressive. However, even if this
EMG-based system is more invasive compared to video or audio systems, it is less invasive compared to previously developed sEMG systems, and it could be used either as standalone wearable system or as an auxiliary system to detect and classify laughter together with audio or video systems. In the future, we are planning to find the causes of misclassification as well as refine the classification algorithm to improve its sensitivity, possibly using machine learning techniques to dynamically adjust the classification depending on individual laughter features.

\section{ACKNOWLEDGMENT}

The authors would like to express their gratitude to the Italian Ministry of Foreign Affairs, General Directorate for Cultural Promotion and Cooperation, for its support to RoboCasa, Asahi Kasei Fibers Corporation, and LP - Life Performance Research. The data collection and design protocol was supported by the Ilhaire grant (FP7/2007-2013) under grant agreement no. 70780. Also, the authors would like to thank Harry Griffin, Hane Aung, and Alessandro Baffa, for their contribution in data acquisition, labelling and analysis.

\section{REFERENCES}

[1] R. R. Provine, Laughter: A Scientific Investigation. Viking, 2000.

[2] W. F. Fry, Sweet madness. Transaction Publishers, 2010.

[3] R. A. Martin, The Psychology of Humor: An Integrative Approach. Academic Press, 2010.

[4] A. Agarwal, D. Nag, and A. K. Jain, Pathological Laughter After Right Hemispheric Infarction.

[5] T. Azuma, A. Shimizu, K. Yamashita, M. Iwase, O. KAJIMOTO, Yoshih. TATSUMOTO, and N. SUMITSUJI, "The inappropriate smile and zygomatic muscle activity", Percept. Mot. Skills, vol. 96, no. 2, pp. 481-491, 2003.

[6] M. P. Bennett and C. Lengacher, "Humor and Laughter May Influence Health: III. Laughter and Health Outcomes", Evid. Based Complement. Alternat. Med., vol. 5, no. 1, pp. 37-40, 2008.

[7] M. H. Brutsche, P. Grossman, R. E. Muller, J. Wiegand, Pello, F. Baty, and W. Ruch, "Impact of laughter on air trapping in severe chronic obstructive lung disease", Int. J. Chron. Obstruct. Pulmon. Dis., vol. 3, no. 1, pp. 185-192, Mar. 2008.

[8] R. R. Provine, Curious Behavior: Yawning, Laughing, Hiccupping, and Beyond. Harvard University Press, 2012.

[9] S. A. Guillory and K. A. Bujarski, "Exploring emotions using invasive methods: review of 60 years of human intracranial electrophysiology", Soc. Cogn. Affect. Neurosci., Feb. 2014.

[10] R. Niewiadomski, M. Mancini, Y. Ding, C. Pelachaud, and G. Volpe, "Rhythmic Body Movements of Laughter", 2014, pp. 299-306.

[11] A. Kleinsmith and N. Bianchi-Berthouze, "Affective Body Expression Perception and Recognition: A Survey", IEEE Trans. Affect. Comput., vol. 4, no. 1, pp. 15-33, Jan. 2013.

[12] K. Hogan, Can't Get Through: Eight Barriers to Communication. Pelican Publishing.

[13] W. Ruch and P. Ekman, "The expressive pattern of laughter", Emot. Qualia Conscious., pp. 426-443, 2001

[14] L. S. Kennedy and D. P. Ellis, "Laughter detection in meetings", in NIST ICASSP 2004 Meeting Recognition Workshop, Montreal, 2004, pp. 118 121.

[15]K. P. Truong and D. A. Van Leeuwen, "Automatic detection of laughter", in In Proc 9th European Conference on Speech Communication and Technology (INTERSPEECH, pp. 485-488. 
[16] K. P. Truong and D. A. van Leeuwen, "Automatic discrimination between laughter and speech", Speech Commun., vol. 49, no. 2, pp. 144 158, Feb. 2007.

[17] M. Knox, “Automatic laughter detection”, Final Proj. EECS 294, 2006.

[18] M. T. Knox and N. Mirghafori, "Automatic laughter detection using neural networks.”, in INTERSPEECH, 2007, pp. 2973-2976.

[19] M. Knox, "Improving Frame Based Automatic Laughter Detection", 2007.

[20] M. T. Knox, N. Morgan, and N. Mirghafori, "Getting the last laugh: Automatic laughter segmentation in meetings," in Proc. INTERSPEECH, 2008, pp. 797-800.

[21] K. Laskowski and S. Burger, "Analysis of the occurrence of laughter in meetings," in INTERSPEECH, 2007, pp. 1258-1261.

[22] T. Jacykiewicz and F. Ringeval, "AUTOMATIC RECOGNITION OF LAUGHTER USING VERBAL AND NON-VERBAL ACOUSTIC FEATURES", Université de Fribourg, 2014.

[23] H. Griffin, M. Aung, B. Romera-Paredes, C. McLoughlin, G. McKeown, W. Curran, and N. Bianchi-Berthouze, "Laughter Type Recognition from Whole Body Motion", in Proceedings of the fifth biannual Humaine Association Conference on Affective Computing and Intelligent Interaction., Geneva, Switzerland, 2013, pp. pp. 349-355.

[24] A. J. Fridlund and J. T. Cacioppo, "Guidelines for Human Electromyographic Research", Psychophysiology, vol. 23, no. 5, pp. 567-589, 1986.

[25] D. Stegeman and H. Hermens, "Standards for surface electromyography: The European project Surface EMG for non-invasive assessment of muscles (SENIAM)", Línea Dispon. En Httpwww Med Uni-Jena Demotorikpdfstegeman Pdf Consult. En Agosto 2008, 2007.

[26] E. Vavrinsky, M. Daricek, M. Donoval, K. Rendek, F. Horinek, M. Horniak, and D. Donoval, "Design of EMG wireless sensor system", in Applied Electronics (AE), 2011 International Conference on, 2011, pp. 1 -4 .

[27] U. Imtiaz, L. Bartolomeo, Z. Lin, S. Sessa, H. Ishii, K. Saito, M. Zecca, and A. Takanishi, "Design of a wireless miniature low cost EMG sensor using gold plated dry electrodes for biomechanics research", in 2013 IEEE International Conference on Mechatronics and Automation (ICMA), 2013, pp. 957-962.

[28] T. A. Olugbade, M. S. H. Aung, N. Marquardt, A. de C. Williams, and N. Bianchi-Berthouze, "Bi-Modal Detection of Painful Reaching for Chronic Pain Rehabilitation Systems".

[29] I. V. E. Huis, G. J. Van Boxtel, and B. de Gelder, "The Body Action Coding System I: muscle activations during the perception and expression of emotion.”, Soc. Neurosci., vol. 9, no. 3, p. 249, 2014.

[30] E. Agostoni, G. Sant"Ambrogio, and H. del Portillo Carrasco, "Electromyography of the diaphragm in man and transdiaphragmatic pressure", J Appl Physiol, vol. 15, no. 6, pp. 1093-1097, 1960.

[31] E. S. Luschei, "Patterns of Laryngeal Electromyography and the Activity of the Respiratory System During Spontaneous Laughter", J. Neurophysiol., vol. 96, no. 1, pp. 442-450, Mar. 2006.

[32] I. R. Titze, E. M. Finnegan, A.-M. Laukkanen, M. Fuja, and H. Hoffman, "Laryngeal Muscle Activity in Giggle: A Damped Oscillation Model", J. Voice, vol. 22, no. 6, pp. 644-648, Nov. 2008.

[33] Y. Kimura and K. Kimura, "Laughter Measurement Method and Apparatus".
[34] H. Kimata, A. Morita, S. Furuhata, H. Itakura, K. Ikenobu, and Y. Kimura, "Assessment of laughter by diaphragm electromyogram", Eur. J. Clin. Invest., vol. 39, no. 1, pp. 78-79, Jan. 2009.

[35] K. P. Strohl, J. Mead, R. B. Banzett, S. H. Loring, and P. C. Kosch, "Regional differences in abdominal muscle activity during various maneuvers in humans", J. Appl. Physiol., vol. 51, no. 6, pp. 1471-1476, 1981.

[36] J. D. Hoit, B. L. Plassman, R. W. Lansing, and T. J. Hixon, "Abdominal muscle activity during speech production”, J. Appl. Physiol., vol. 65, no. 6, pp. 2656-2664, 1988.

[37] S. Fukushima, Y. Hashimoto, T. Nozawa, and H. Kajimoto, "Laugh enhancer using laugh track synchronized with the user's laugh motion", in CHI'10 Extended Abstracts on Human Factors in Computing Systems, 2010, pp. 3613-3618.

[38]S. Cosentino, T. Kishi, M. Zecca, S. Sessa, L. Bartolomeo, K. Hashimoto, T. Nozawa, and A. Takanishi, "Human-humanoid robot social interaction: Laughter", in 2013 IEEE International Conference on Robotics and Biomimetics (ROBIO), 2013, pp. 1396-1401.

[39] G. McKeown, W. Curran, C. McLoughlin, H. J. Griffin, and N. BianchiBerthouze, "Laughter induction techniques suitable for generating motion capture data of laughter associated body movements", in 2013 10th IEEE International Conference and Workshops on Automatic Face and Gesture Recognition (FG), 2013, pp. 1-5.

[40] G. A. Bryant and C. A. Aktipis, "The animal nature of spontaneous human laughter", Evol. Hum. Behav., vol. 35, no. 4, pp. 327-335, Jul. 2014.

[41] G. McKeown, I. Sneddon, and W. Curran, "Gender differences in the perceptions of genuine and simulated laughter and amused facial expressions", Emot. Rev., vol. 7, no. 1, pp. 30-38, 2015.

[42] B. Hudgins, P. Parker, and R. N. Scott, "A new strategy for multifunction myoelectric control", IEEE Trans. Biomed. Eng., vol. 40, no. 1, pp. 82-94, Jan. 1993.

[43] I. H. Witten, E. Frank, and M. A. Hall, Data Mining: Practical Machine Learning Tools and Techniques, Third Edition, 3 edition. Burlington, MA: Morgan Kaufmann, 2011.

[44] H. Kimata, A. Morita, S. Furuhata, H. Itakura, K. Ikenobu, and Y. Kimura, "Assessment of laughter by diaphragm electromyogram", Eur. J. Clin. Invest., vol. 39, no. 1, pp. 78-79, 2009.

[45] S. Fukushima, Y. Hashimoto, T. Nozawa, and H. Kajimoto, "Laugh enhancer using laugh track synchronized with the user's laugh motion", in CHI'10 Extended Abstracts on Human Factors in Computing Systems, 2010, pp. 3613-3618.

[46] Y. Kimura and K. Kimura, "Laughter Measurement Method and Apparatus".

[47] 板村英典, 池田資尚, 池信敬子, and 森下伸也, “筋電計を用いた「笑 い」の分類と定量化システムの検討”, 人間健康学研究, vol. 4, pp. 79-90, 2012.

[48] 池田資尚, 板村英典, 池信敬子, and 森下伸也, “顔・喉・腹の「3 点 計測システム」による「笑い」の客観的分類法の検討”, RIDEO, no. 19, pp. 75-85, Jul. 2012.

[49] T. Pothirat, S. Chatpun, P. Phukpattaranont, and D. Vongjandaeng, "The optimal electromyography feature for oral muscle movements", in Biomedical Engineering International Conference (BMEiCON), 2013 6th, 2013, pp. 1-5. 